\title{
Rational points on abelian varieties over function fields and Prym varieties
}

\author{
ABolfazl MOHAJeR(D
}

\begin{abstract}
In this paper, using a generalization of the notion of Prym variety for covers of projective varieties, we prove a structure theorem for the Mordell-Weil group of abelian varieties over function fields that are twists of abelian varieties by Galois covers of smooth projective varieties. In particular, the results we obtain contribute to the construction of Jacobians of high rank.
\end{abstract}

Mathematics Subject Classification. 14G05, 14H30, $14 \mathrm{H} 40$.

Keywords. Prym variety, Rational points, Galois covering.

1. Introduction. Let $A$ be an abelian variety over a field $k$ of characteristic $\geq 0$. The Mordell-Weil theorem asserts that if $k$ is furthermore a number field, then the set of $k$-rational points $A(k)$ on $A$ is a finitely generated abelian group. The rank of this abelian group is referred to as the rank of the abelian variety $A$ with notation $\operatorname{rank}(A(k))$. One interesting problem in arithmetic geometry is to find abelian varieties with arbitrarily large ranks.

The present paper is a generalization of the work of Salami in [5] to arbitrary Galois coverings. More precisely, let $n \in \mathbb{N}$ be a natural number such that the characteristic of the field $k$ in the previous paragraph does not divide $n$ and $k$ contains a primitive $n$-th root of unity $\xi$. Suppose there is an embedding $G \hookrightarrow \operatorname{Aut}(A)$ with $G$ a finite group with $|G|=n$. Further, let $X, Y$ be smooth projective varieties over $k$ with function fields $F, K$ respectively. We first define the notion of Prym variety associated to a $G$-Galois covering $f: X \rightarrow Y$. We will show that this is a natural generalization of the notion of Prym variety in the case of coverings of curves. The group $G$ is well-known to be also the Galois group of the Galois field extension $F / K$. By the results of $[3,4]$, the twist of $A$ by the extension $F / K$ is equivalent to a twist by the 1-cocyle $a=\left(a_{g}\right) \in Z^{1}(G, \operatorname{Aut}(A))$ given by $a_{g}=g$, where $g$ is viewed both as a group 
element and as an automorphism of $A$ corresponding to $g \in G$ (in other words, we identify $g$ with its image $g \in G \hookrightarrow \operatorname{Aut}(A))$. We prove a theorem which gives an isomorphism for $A_{a}(K)$ in terms of the Prym variety introduced in Section 2, see Theorem 2.4. Then we consider the $n$-times product $\prod_{i} f$ of a $G$-cover $f: X \rightarrow Y$ with itself. We prove a result which describes the Prym variety of the self product of $G$-covers of varieties in terms of the Prym variety of the cover. We apply this to the case of an abelian cover $C \rightarrow \mathbb{P}^{1}$. More precisely, we prove that $\operatorname{rank}\left(\operatorname{Jac}(C)_{a}(K)\right)$ can be made arbitrarily large by taking $n$ large enough.

2. The Prym variety of a Galois covering. Let $V$ be a smooth projective variety over a field $k$. The Albanese variety of $V$ is by definition the initial object for the morphisms from $V$ to abelian varieties. In particular, if $V$ is moreover defined over a field of characteristic zero (the classical case), it is the abelian variety

$$
\operatorname{Alb}(V)=H^{0}\left(V, \Omega_{V}^{1}\right)^{*} / H_{1}(V, \mathbb{Z}) .
$$

Now let $X, Y$ be smooth projective varieties with $f: X \rightarrow Y$ a Galois covering with the Galois group $G$. Throughout this paper, we consider the situation where there is a finite group $G$ with $|G|=n$ that has a $G$-action on $X$ such that $Y:=X / G$ and $f: X \rightarrow Y$ is the quotient map. The universal property of the Albanese variety implies that there is an induced action of $G$ on $\operatorname{Alb}(X)$. We denote by $\left(\operatorname{Alb}(X)^{G}\right)^{0}$ the largest abelian subvariety of $\operatorname{Alb}(X)$ fixed (pointwise) under this action. Equivalently, $\left(\operatorname{Alb}(X)^{G}\right)^{0}$ is the connected component containing the identity of the subvariety $\operatorname{Alb}(X)^{G}$ of fixed points of $\operatorname{Alb}(X)$ under the action of $G$. In characteristic zero, the induced action of $G$ on $\operatorname{Alb}(X)$ can be described through its action on $H^{0}\left(X, \Omega_{X}^{1}\right)$. With respect to this action, we write

$$
\begin{aligned}
& H^{0}\left(X, \Omega_{X}^{1}\right)^{+}=H^{0}\left(X, \Omega_{X}^{1}\right)^{G}\left(\cong H^{0}\left(Y, \Omega_{Y}^{1}\right)\right) \text { and } \\
& H^{0}\left(X, \Omega_{X}^{1}\right)^{-}=H^{0}\left(X, \Omega_{X}^{1}\right) / H^{0}\left(X, \Omega_{X}^{1}\right)^{+}=\bigoplus_{\chi \in \operatorname{Irr}(G) \backslash\{1\}} H^{0}\left(\widetilde{C}, \omega_{\widetilde{C}}\right)^{\chi} .
\end{aligned}
$$

Similarly, one defines $H_{1}(X, \mathbb{Z})^{-}$. Here $\operatorname{Irr}(G)$ is the set of irreducible characters of $G$.

Let $g \in G$ be an element of the group $G$. We will denote also by $g$ the automorphism of $\operatorname{Alb}(X)$ induced by $g$. Using this notation and following that of [8, Prop. 3.1] for the case of curves, we write $\operatorname{Nm} G: \operatorname{Alb}(X) \rightarrow \operatorname{Alb}(X)$ for the norm endomorphism of $\operatorname{Alb}(X)$ given by $\operatorname{Nm} G:=\sum_{g \in G} g$.

Now we are ready to define the notion of Prym variety for the covering $f$.

Definition 2.1. Let $f: X \rightarrow Y$ be a $G$-Galois covering of smooth projective varieties over a field $k$. The Prym variety $P(X / Y)$ associated with the covering $f$ is defined to be

$$
P(X / Y):=\frac{\operatorname{Alb}(X)}{\left(\operatorname{Alb}(X)^{G}\right)^{0}}
$$


For the classical case of Prym varieties of double coverings of curves, we refer to [1]. The following result gives an equivalent description of the Prym variety and shows furthermore that it coincides with the Prym variety of covers of curves (see [1,6-8]) up to isogeny.

Proposition 2.2. With notation and assumptions of Definition 2.1, it holds that $P(X / Y)=\frac{\operatorname{Alb}(X)}{\operatorname{Im} \mathrm{Nm} G}$. Furthermore it is isogeneous to the abelian variety $(\operatorname{ker} \mathrm{Nm} G)^{0}$. In particular, if the varieties are defined over a field of characteristic zero, then $P(X / Y)$ is isogeneous to the abelian variety $\operatorname{Alb}(X)^{-}=$ $\left(H^{0}\left(X, \Omega_{X}^{1}\right)^{-}\right)^{*} / H_{1}(X, \mathbb{Z})^{-}$.

Proof. The induced linear action on the tangent space of $\operatorname{Alb}(X)$ at the origin gives that $\operatorname{dim} \operatorname{Alb}(X)=\operatorname{dim}(\operatorname{kerNm} G)^{0}+\operatorname{dim}\left(\operatorname{Alb}(X)^{G}\right)^{0}$. Let $Q \in$ $\left(\operatorname{Alb}(X)^{G}\right)^{0} \cap(\operatorname{kerNm} G)^{0}$. It follows that $n Q=0$ in $\operatorname{Alb}(X)$, therefore $\left(\operatorname{Alb}(X)^{G}\right)^{0} \cap(\operatorname{ker} \mathrm{Nm} G)^{0} \subseteq \operatorname{Alb}(X)[n]$. In particular, this intersection is finite so that $\operatorname{Alb}(X)$ is isogenous to the product $\left(\operatorname{Alb}(X)^{G}\right)^{0} \times(\operatorname{ker} \operatorname{Nm} G)^{0}$. Since $\left(\sum_{g \in G} g\right) \cdot h=\sum_{g \in G}(g \cdot h)=\sum_{g \in G} g$ for every $h \in G$, it follows that $\operatorname{Im} \operatorname{Nm} G \subseteq\left(\operatorname{Alb}(X)^{G}\right)^{0}$. On the other hand, the above isogeny decomposition for $\operatorname{Alb}(X)$ implies that $\operatorname{dim} \operatorname{Im} \mathrm{Nm} G=\operatorname{dim} \operatorname{Alb}(X)-\operatorname{dim}(\operatorname{ker} \mathrm{Nm} G)^{0}=$ $\operatorname{dim}\left(\operatorname{Alb}(X)^{G}\right)^{0}$. Hence $\operatorname{Im~Nm} G=\left(\operatorname{Alb}(X)^{G}\right)^{0}$. This completes the proof of the claims.

Remark 2.3. For curves, the Albanese variety coincides with the Jacobian variety. Hence if $X$ and $Y$ are curves, the Prym variety $P(X / Y)$ coincides with the Prym variety for covers of curves, see $[6,7]$. In this case, there are two fundamental homomorphisms: the norm homomorphism $\mathrm{Nm}_{f}: \operatorname{Jac}(X) \rightarrow \operatorname{Jac}(Y)$ and the pull-back homomorphism $f^{*}: \operatorname{Jac}(Y) \rightarrow \operatorname{Jac}(X)$ and it holds that $\mathrm{Nm} G=f^{*} \circ \mathrm{Nm}_{f}$, so that $P(X / Y)=\left(\operatorname{ker} \mathrm{Nm}_{f}\right)^{0}=(\operatorname{ker} \mathrm{Nm} G)^{0}$, see $[8$, Prop. 3.1].

We recall from $[3,4]$ the notion of twist of a smooth projective variety. Suppose $k^{\prime} / k$ is a $G$-Galois extension of fields. Let $\mathcal{A}$ be a $G$-set and suppose $E$ is another $G$-set which is also a left $\mathcal{A}$-set. Let $a=\left(a_{g}\right) \in Z^{1}(G, \operatorname{Aut}(\mathcal{A}))$ be a 1-cocycle of $\mathcal{A}$. For any $g \in G$ and $x \in E$, we denote by ${ }^{g} x$ the left action of $g$ on $x$. The $G$-set $E$ with this action of $G$ is denoted by $E_{a}$ and is called the twist of $E$ by $a$. If $X$ is a projective smooth variety over $k$, we denote by $\operatorname{Aut}(X)$ the automorphism scheme of $X$ and let $a=\left(a_{g}\right) \in Z^{1}(G$, Aut $(X))$. There exist a projective variety $Y$ over $k$ and a $k^{\prime}$-isomorphism $f^{\prime}: X \otimes_{k} k^{\prime} \rightarrow Y \otimes_{k} k^{\prime}$ such that ${ }^{g}\left(f^{\prime}\right)=f^{\prime} \circ a_{g}$ for every $g \in G$. We denote the variety $Y$ by $X_{a}$ and call it the twist of $X$ by $a$. Of course the two notions of twist introduced above are compatible in the sense that the map $f^{\prime}: X\left(k^{\prime}\right) \rightarrow Y\left(k^{\prime}\right)$ induces an isomorphism between $X\left(k^{\prime}\right)_{a} \cong X_{a}\left(k^{\prime}\right)\left(=Y\left(k^{\prime}\right)\right)$.

In this paper, the above results will be applied to an abelian variety $A$ and its twist $A_{a}$ by the extension $F / K$ or equivalently by the 1-cocycle $a=\left(a_{g}\right) \in$ $Z^{1}(G, \operatorname{Aut}(A))$ given by $a_{g}=g$. 
Theorem 2.4. Assume that there exists a $k$-rational point $x_{0} \in X(k)$. Then there is an isomorphism of abelian groups

$$
A_{a}(K) \cong \operatorname{Hom}_{k}(P(X / Y), A) \oplus A[n](k) .
$$

If in addition $P(X / Y)$ is k-isogenous to $A^{n} \times B$, where $n \in \mathbb{N}$ and $B$ is an abelian variety over $k$ that does not have any simple components $k$-isogenous to $A$, then $\operatorname{rank}\left(A_{a}(K)\right)=n \cdot \operatorname{rank}\left(\operatorname{End}_{k}(A)\right)$.

Proof. Note that the universal property of the Albanese variety together with the fact that a regular morphism of abelian varieties is obtained by a homomorphism followed by a translation (see, for example, [2, Prop. 1.2.1]) shows that

$$
A(K) \cong \operatorname{Hom}_{k}(\operatorname{Alb}(X), A) \oplus A(k) .
$$

We assume that the Albanese map $\iota_{X}: X \rightarrow \operatorname{Alb}(X)$ satisfies $\iota_{X}\left(x_{0}\right)=0$ so that it is defined over $k$. Recall that [4, Prop. 1.1] shows that

$$
A_{a}(K) \cong\left\{P \in A(F) \mid a_{g} \cdot{ }^{g}(P)=P\right\} .
$$

This implies in our particular case that for any $g \in G$, viewed as remarked earlier also as an automorphism of $\operatorname{Alb}(X)$, we have that $(\alpha, Q) \in A_{a}(K)$ if and only if $g(\alpha \circ g, Q)=(\alpha, Q)$ or equivalently $(\alpha \circ g, Q)=g^{-1}(\alpha, Q)$. But this is the case if and only if $\alpha$ annihilates $\operatorname{Im} \operatorname{Nm} G$ and $Q \in A[n](k)$. Proposition 2.2 then shows that it must actually lie in $P(X / Y)$, so we obtain the claimed isomorphism in (2.4.1).

If moreover $P(X / Y)$ is isogenous to $A^{n} \times B$ with $n, A$, and $B$ as in the statement of the proposition, then

$$
\begin{aligned}
A_{a}(K) & \cong \operatorname{Hom}_{k}(P(X / Y), A) \oplus A[n](k) \\
& \cong \operatorname{Hom}_{k}\left(A^{n} \times B, A\right) \oplus A[n](k) \\
& \cong \operatorname{Hom}_{k}\left(A^{n}, A\right) \oplus \operatorname{Hom}_{k}(B, A) \oplus A[n](k) \\
& \cong \operatorname{End}_{k}(A)^{n} \oplus \operatorname{Hom}_{k}(B, A) \oplus A[n](k) .
\end{aligned}
$$

Which implies that as $\mathbb{Z}$-modules, it holds that $\operatorname{rank}\left(A_{a}(K)\right)=$ $n \cdot \operatorname{rank}\left(\operatorname{End}_{k}(A)\right)$.

Given a $G$-Galois covering $f: X \rightarrow Y$, one can form the $n$-fold self product $\prod_{i=1}^{n} f: \prod_{i=1}^{n} X \rightarrow \prod_{i=1}^{n} Y$ which is a $\underbrace{G \times \cdots \times G}_{n-\text { times }}$ - Galois covering. Then the diagonal embedding $G \hookrightarrow \prod_{i} G:=G \times \cdots \times G$ gives a subgroup of $\prod_{i} G$ isomorphic to $G$. We denote this subgroup by $\tilde{G}$. This gives rise to an intermediate Galois covering $f: \prod_{i=1}^{n} X \rightarrow\left(\prod_{i=1}^{n} X\right) / \tilde{G}$. Let us write $\mathcal{X}=\prod_{i=1}^{n} X$ and $\mathcal{Y}=\left(\prod_{i} X\right) / \tilde{G}$. We are interested in the Prym variety $P(\mathcal{X} / \mathcal{Y})$. In fact, we show

Proposition 2.5. With the above notation, there is an isogeny

$$
P(\mathcal{X} / \mathcal{Y}) \sim_{k} \prod_{i} P\left(X_{i} / Y_{i}\right)
$$


Proof. It suffices to only treat the case $n=2$. The general case follows by an induction argument. So suppose $n=2$ and denote the Galois group of the cover $\mathcal{X} / \mathcal{Y}$ by $\tilde{G}(\cong G)$. By Proposition 2.2 , it suffices to show that there is a $k$-isogeny $(\operatorname{ker} \operatorname{Nm} \tilde{G})^{0} \sim_{k}(\operatorname{ker} \operatorname{Nm} G)^{0} \times(\operatorname{ker} \operatorname{Nm} G)^{0}$. In fact, we show that there is an isomorphism between these abelian varieties. Notice that there is an isomorphism

$$
\beta: \operatorname{Alb}\left(X_{1}\right) \times \operatorname{Alb}\left(X_{2}\right) \stackrel{\sim}{\longrightarrow} \operatorname{Alb}(\mathcal{X}) .
$$

The isomorphism $\beta$ is given as follows: Let $j_{i}: X_{i} \rightarrow \mathcal{X}$, for $i=1,2$, be the natural inclusions. Then $\beta=\tilde{j_{1}}+\tilde{j_{2}}$, where $\tilde{j}_{i}$ denotes the induced homomorphism $\operatorname{Alb}\left(X_{i}\right) \rightarrow \operatorname{Alb}(\mathcal{X})$. This isomorphism is compatible with the action of $\tilde{G}$, namely, there is the following commutative diagram. From this, one deduces the isomorphism $\operatorname{ker} \mathrm{Nm} \tilde{G} \stackrel{\sim}{\longrightarrow} \operatorname{ker} \mathrm{Nm} G \times \operatorname{ker} \mathrm{Nm} G$ which implies the desired isomorphism.

Consider an abelian cover $C \rightarrow \mathbb{P}^{1}$ with Galois group $G$. Consider the product $\mathcal{C}_{n}=\prod_{i=1}^{n} C$, i.e., the product of $n$ copies of the same abelian cover in the above and let $\tilde{G}$ be the image of $G$ under the diagonal embedding $G \hookrightarrow \prod_{i=1}^{n} G$ as above. Set $\mathcal{D}_{n}=\mathcal{C}_{n} / \tilde{G}$. By Proposition 2.5 , we have that

$$
P\left(\mathcal{C}_{n} / \mathcal{D}_{n}\right)=\prod_{i} P\left(C / \mathbb{P}^{1}\right)
$$

By Remark 2.3, $P\left(C / \mathbb{P}^{1}\right)=\left(\operatorname{ker} \mathrm{Nm}_{f}\right)^{0}$. However, as $\operatorname{Jac}\left(\mathbb{P}^{1}\right)=0$, it follows that $P\left(C / \mathbb{P}^{1}\right)=\operatorname{Jac}(C)$. Now Proposition 2.5 gives that

$$
P\left(\mathcal{C}_{n} / \mathcal{D}_{n}\right)=\prod_{i} P\left(C / \mathbb{P}^{1}\right)=(\operatorname{Jac}(C))^{n} .
$$

Note that the function field $K(C)$ of $C$ is generated over the function field $K\left(\mathbb{P}^{1}\right)=K(z)$ of $\mathbb{P}^{1}$ by taking roots of (transcendental) elements of $K(C)$, i.e., it is of the form $K(z)\left(x_{1}^{1 / m}, \ldots, x_{r}^{1 / m}\right)$. Then the function field $\mathcal{L}_{n}$ of $\mathcal{C}_{n}$ is $K(z)\left(x_{i 1}^{1 / m}, \ldots, x_{i r}^{1 / m}\right), i=1, \ldots, n$. Let $K=k\left(\mathcal{D}_{n}\right)$ be the function field of $\mathcal{D}_{n}$.

We define the 1-cocycle $Z^{1}(G$, Aut $(C))$ by $a_{g}=g$. Let $\operatorname{Jac}(C)_{a}$ be the twist corresponding to this 1-cocycle. By applying (2.4.1) and (2.5.4), it follows that

$$
\begin{aligned}
\operatorname{Jac}(C)_{a}(K) & \cong \operatorname{Hom}_{k}\left(P\left(\mathcal{C}_{n} / \mathcal{D}_{n}\right), \operatorname{Jac}(C)\right) \oplus \operatorname{Jac}(C)[n](k) \\
& \cong \operatorname{Hom}_{k}\left((\operatorname{Jac}(C))^{n}, \operatorname{Jac}(C)\right) \oplus \operatorname{Jac}(C)[n](k) \\
& \cong \operatorname{End}_{k}(\operatorname{Jac}(C))^{n} \oplus \operatorname{Jac}(C)[n](k)
\end{aligned}
$$

So that $\operatorname{rank}\left(\operatorname{Jac}(C)_{a}(K)\right)=n \cdot \operatorname{rank}\left(\operatorname{End}_{k}(\operatorname{Jac}(C))\right)$. Let us see an example.

Example 2.6. An abelian Galois cover $C \rightarrow \mathbb{P}^{1}$ is determined by a collection of equations in the following way:

Consider an $m \times s$ matrix $A=\left(r_{i j}\right)$ whose entries $r_{i j}$ are in $\mathbb{Z} / N \mathbb{Z}$ for some $N \geq 2$. Let $\overline{k(z)}$ be the algebraic closure of $k(z)$. For each $i=1, \ldots, m$, choose 
a function $w_{i} \in \overline{k(z)}$ with

$$
w_{i}^{N}=h_{i}(z)=\prod_{l=1}^{s}\left(z-z_{l}\right)^{\widetilde{r}_{l i}} \text { for } i=1, \ldots, m .
$$

Here $\widetilde{r}_{i j}$ is the lift of $r_{i j}$ to $\mathbb{Z} \cap[0, N)$ and $z_{j} \in k$ for $j=1,2, \ldots, s$. Notice that (2.6.1) gives in general only a singular affine curve and we take a smooth projective model associated to this affine curve. The Galois group $\tilde{G}$ of the covering is a subgroup of $(\mathbb{Z} / N \mathbb{Z})^{m}$. We will assume for simplicity that $\tilde{G}=$ $(\mathbb{Z} / N \mathbb{Z})^{m}$. This is the case if the rows of the matrix are linearly independent over $\mathbb{Z} / N \mathbb{Z}$, for example if $N$ is a prime number. Now consider the $n$-fold product $\mathcal{C}_{n}$ as introduced above. Denoting the independent transcendental variables on the copy $C_{i}$ of $C$ by $z_{i}$ and $w_{i j}(j=1, \ldots, m)$, we see that the function field of $\mathcal{C}_{n}$ is $L=k\left(z_{1}, \ldots, z_{n}, w_{i j}\right)(i=1, \ldots, n, j=1, \ldots, m)$. The branch points of each copy $C_{i}$ as in (2.6.1) will be denoted by $z_{i l}, l=$ $1, \ldots, s$. Then the function field $K=k\left(\mathcal{D}_{n}\right)$ of $\mathcal{D}_{n}$ is the set of $\tilde{G}$-invariant elements of $L$ under the action of $\tilde{G}$, i.e., $K=k\left(z_{1}, \ldots, z_{n}, w_{i j}\right)^{\tilde{G}}$. In this case, $K=k\left(z_{1}, \ldots, z_{n}, w_{1 j}^{N-1} w_{i j}\right)(i \neq 1)$. Since $\left(w_{1 j}^{N-1} w_{i j}\right)^{N}=\left(\prod_{l=1}^{s}\left(z_{1}-\right.\right.$ $\left.\left.z_{1 l}\right)^{\widetilde{r}_{l j}}\right)^{N-1} \prod_{l=1}^{s}\left(z_{i}-z_{i l}\right)^{\widetilde{r}_{i j}}, j=1, \ldots, m$, by setting $W_{i j}=w_{1 j}^{N-1} w_{i j}$, one obtains a new abelian cover with function field $K$ such that the extension $L=K\left(w_{1 j} \mid j=1, \ldots, m\right)$ is also abelian with Galois group $\tilde{G}$. So we can also write $L=k\left(z_{1}\right)\left(u_{1}, \ldots, u_{m}\right)$ where $u_{i}=\sqrt[N]{h_{i}\left(z_{1}\right)}$ for $i=1, \ldots, m\left(h_{i}\right.$ as in (2.6.1)). Suppose $C_{a}$ is the twist of the abelian cover $C$ by the extension $L \mid K$. Arguing as in [4, Corollary 3.1], one sees that $C_{a}$ can be defined by the equation $\prod_{l=1}^{s}\left(z_{1}-z_{1 l}\right)^{\widetilde{r}_{i j}} W_{i}^{N}=\prod_{l=1}^{s}\left(z-z_{j}\right)^{\widetilde{r}_{i j}}, i=1, \ldots, m$, or in the notation of (2.6.1), $h_{i}\left(z_{1}\right) W_{i}^{N}=h_{i}(z)$. Let us show this in detail. Let $D$ denote the curve over $k\left(z_{1}\right)$ defined by the equation $h_{i}\left(z_{1}\right) W_{i}^{N}=h_{i}(z), i=1, \ldots, m$, for each $1 \leq i \leq m$. Define $f^{\prime}: C \otimes_{k} L \rightarrow D \otimes_{K} L$ by

$$
\begin{aligned}
& \left(z\left(z_{1}, u\right), w_{1}\left(z_{1}, u\right), \ldots, w_{i}\left(z_{1}, u\right), \ldots, w_{m}\left(z_{1}, u\right)\right) \\
& \quad \mapsto\left(z\left(z_{1}, u\right), w_{1}\left(z_{1}, u\right) / u_{1}, \ldots, w_{i}\left(z_{1}, u\right) / u_{i}, \ldots, w_{m}\left(z_{1}, u\right) / u_{m}\right)
\end{aligned}
$$

where we use $\left(z_{1}, u\right)$ as an abbreviation for the coordinate $\left(z_{1}, u_{1}, \ldots, u_{m}\right)$ in $L=k\left(z_{1}\right)\left(u_{1}, \ldots, u_{m}\right)$ as mentioned above. Suppose $\tilde{G}=\left\langle g_{1}\right\rangle \times \cdots \times\left\langle g_{m}\right\rangle$. The action of $\tilde{G}$ on $L$ is by letting $g_{i}$ act as $g_{i} \cdot\left(z_{1}, u\right)=\left(z_{1}, u_{1}, \ldots, \xi u_{i}, \ldots, u_{m}\right)$, where $\xi$ is an $N$-th primitive root of unity, whose existence was assumed from the beginning of this paper in Section 1. With the above notation, we have

$$
\begin{aligned}
g_{i} & f^{\prime}\left(z\left(z_{1}, u\right), w_{1}\left(z_{1}, u\right), \ldots, w_{m}\left(z_{1}, u\right)\right) \\
& ={ }^{g_{i}}\left(f^{\prime}\left({ }^{g_{i}}{ }^{N-1}\left(z\left(z_{1}, u\right), w_{1}\left(z_{1}, u\right), \ldots, w_{m}\left(z_{1}, u\right)\right)\right)\right) \\
& ={ }^{g_{i}}\left(f^{\prime}\left(z\left(z_{1}, u_{1}, \ldots, \xi^{N-1} u_{i}, \ldots, u_{m}\right), \ldots, w_{m}\left(z_{1}, u_{1}, \ldots, \xi^{N-1} u_{i}, \ldots, u_{m}\right)\right)\right) \\
& ={ }^{g_{i}}\left(z\left(z_{1}, u_{1}, \ldots, \xi^{N-1} u_{i}, \ldots, u_{m}\right), \ldots, w_{m}\left(z_{1}, u_{1}, \ldots, \xi^{N-1} u_{i}, \ldots, u_{m}\right) / u_{m}\right) \\
& =\left(z\left(z_{1}, u\right), \xi w_{1}\left(z_{1}, u\right) / u_{1}, \ldots, \xi w_{m}\left(z_{1}, u\right) / u_{m}\right) .
\end{aligned}
$$


Note that the first equality is due to the fact that $g_{i}{ }^{N}=1$. On the other hand, we have

$$
\begin{aligned}
f^{\prime} & \circ a_{g_{i}}\left(z\left(z_{1}, u\right), w_{1}\left(z_{1}, u\right), \ldots, w_{m}\left(z_{1}, u\right)\right) \\
& =f^{\prime}\left(z\left(z_{1}, u\right), \xi w_{1}\left(z_{1}, u\right), \ldots, \xi w_{i}\left(z_{1}, u\right), \ldots, \xi w_{m}\left(z_{1}, u\right)\right) \\
& =\left(z\left(z_{1}, u\right), \xi w_{1}\left(z_{1}, u\right) / u_{1}, \ldots, \xi w_{m}\left(z_{1}, u\right) / u_{m}\right) .
\end{aligned}
$$

Therefore we have the equality ${ }^{g_{i}} f^{\prime}=f^{\prime} \circ a_{g_{i}}$ for every $i=1, \ldots, m$. Since the $g_{i}$ are generators of $G$, it holds that ${ }^{g} f^{\prime}=f^{\prime} \circ a_{g}$ for every $g \in G$ which implies the isomorphism $D \cong C_{a}$ because of the uniqueness of the twist by [3, Prop. 2.6]. So $C_{a}$ contains the rational points $P_{1}=\left(z_{1}, 1\right), P_{i}=\left(z_{i+1, j}, W_{i j} / \prod_{l=1}^{s}\left(z_{1}-\right.\right.$ $\left.\left.z_{1 j}\right)^{\widetilde{r}_{i j}}\right)(1 \leq i \leq n-1)$. By the above computations, we see that

$$
\operatorname{Jac}(C)_{a}(K) \cong \operatorname{End}_{k}(\operatorname{Jac}(C))^{n} \oplus \operatorname{Jac}(C)[n](k) .
$$

Denote by $Q_{i}$ the image of $P_{i}$ via the Abel-Jacobi embedding $C_{a} \hookrightarrow J\left(C_{a}\right)$. Then the $Q_{i}$ are among the generators of $\operatorname{Jac}(C)_{a}(K)$.

Acknowledgements. I acknowledge the referee for helpful comments and suggestions that significantly improved the paper and also the editor for his efforts on the submission.

Funding Open Access funding enabled and organized by Projekt DEAL.

Open Access. This article is licensed under a Creative Commons Attribution 4.0 International License, which permits use, sharing, adaptation, distribution and reproduction in any medium or format, as long as you give appropriate credit to the original author(s) and the source, provide a link to the Creative Commons licence, and indicate if changes were made. The images or other third party material in this article are included in the article's Creative Commons licence, unless indicated otherwise in a credit line to the material. If material is not included in the article's Creative Commons licence and your intended use is not permitted by statutory regulation or exceeds the permitted use, you will need to obtain permission directly from the copyright holder. To view a copy of this licence, visit http://creativecommons. org/licenses/by/4.0/.

Publisher's Note Springer Nature remains neutral with regard to jurisdictional claims in published maps and institutional affiliations.

\section{References}

[1] Beauville, A.: Variétiés de Prym et Jacobiennes intermediares. Ann. Scient. Éc. Norm. Sup. 10, 309-391 (1977)

[2] Birkenhake, C., Lange, H.: Complex Abelian Varieties. Springer, New York (1992)

[3] Borel, A., Serre, J.-P.: Théorèmes de finitude en cohomologie galoisienne. Comment. Math. Helv. 39, 111-164 (1964)

[4] Hazama, F.: On the Mordell-Weil group of certain abelian varieties defined over function fields. J. Number Theory 37, 168-172 (1991) 
[5] Hazama, F.: Rational points on certain abelian varieties over the rational function field. J. Number Theory 50, 278-285 (1995)

[6] Lange, H., Ortega, A.: Prym varieties of cyclic coverings. Geom. Dedicata. 150, 391-403 (2011)

[7] Mohajer, A.: On the Prym map of Galois coverings. arXiv:2004.09678 (2020)

[8] Recillas, S., Rodríguez, R.: Prym varieties and fourfold covers. arXiv:math/0303155 (2003)

[9] Salami, S.: The rational points on certain abelian varieties over function fields. J. Number Theory 195, 330-337 (2019)

Abolfazl Mohajer

Institut für Mathematik

Johannes Gutenberg Universität Mainz

Staudingerweg 9

55099 Mainz

Germany

e-mail: mohajer@uni-mainz.de

Received: 12 August 2020

Revised: 8 October 2020

Accepted: 23 October 2020. 